\title{
EFFECT OF DELAYED CORD CLAMPING VERSUS CORD MILKING AMONG PRETERM INFANTS: A RANDOMIZED CONTROLLED TRIAL
}

\author{
Tanai Trongkamonthum*, Benjamaporn Puangpaka**, Prisana Panichkul**, Sangkae Chamnanvanakij* \\ * Department of Pediatrics, Phramongkutklao Hospital, Bangkok, Thailand \\ ** Department of Obstetrics and Gynecology, Phramongkutklao Hospital, Bangkok, Thailand
}

\begin{abstract}
Background: Delayed cord clamping (DCC) is currently recommended for preterm infants to improve blood volume and decrease the rate of blood transfusion. Umbilical cord milking $(\mathrm{CM})$ has similar advantages without interrupting neonatal resuscitation. However, the differences in neonatal outcomes between DCC and CM have not been well elucidated.

Objective: To compare neonatal outcomes between DCC and CM among preterm infants Methods: Infants born at 25-34 weeks of gestation were randomly allocated to 1 of 2 groups; group 1 received DCC for 60 seconds whereas group 2 received CM. Initial hemoglobin value was measured, while blood pressure and urine output were monitored. Neonatal complications and the rate of blood transfusion were recorded.

Results: Twenty-two infants were enrolled in each group. No differences were observed regarding sex, gestational age, birth weight, mode of delivery, Apgar scores and rate of resuscitation between groups. The initial hemoglobin level of the DCC group [median $17.1(13.1,21.3) \mathrm{g} / \mathrm{dL}$ ] did not differ from that of the CM group [median $17.1(14.0,22.5)$ $\mathrm{g} / \mathrm{dL}], p=0.963$. During the first 24 hours, no significant differences were observed regarding blood pressure and urine output between groups. No differences were found in the rates of hypothermia, hyperbilirubinemia, intraventricular hemorrhage, necrotizing enterocolitis and rate of blood transfusion.

Conclusion: We demonstrated no different effects on neonatal outcomes between DCC and CM among preterm infants. CM can be applied as an alternative to DCC especially in emergency situations. However, larger studies are warranted to determine the effects and safety of $\mathrm{CM}$ among preterm infants.
\end{abstract}

Keywords : Delayed cord clamping, Umbilical cord milking

J Southeast Asian Med Res 2018; 2(1): 22-27.

http://www.jseamed.org

\section{Correspondence to:}

Chamnanvanakij S, Department of Pediatrics, Phramongkutklao Hospital, Bangkok, Thailand E-mail : chamnanvanakij@gmail.com 


\section{Introduction}

Currently, strong evidence supports delayed cord clamping (DCC) over immediate cord clamping (ICC) among preterm infants. ${ }^{(1)}$ Many studies have confirmed that DCC increased initial hematocrit levels ${ }^{(2-4)}$, improved transitional hemodynamic status ${ }^{(5-7)}$, decreased blood transfusion due to anemia ${ }^{(8,9)}$ and reduced rates of intraventricular hemorrhage. ${ }^{(9-11)}$ Since 2006, many professional societies and committees have recommended DCC for at least 30 seconds at preterm births. ${ }^{(12,13)}$ Umbilical cord milking (UM) was proposed as an alternative to DCC without delayed neonatal resuscitation and was proved to have similar benefits compared with ICC. ${ }^{(14-21)}$ However, UM has not been recommended especially among infants born at less than 29 weeks of gestation due to the issue of rapid changes of blood volume. To date, only a few studies have been conducted comparing neonatal outcomes between DCC and CM among preterm infants. ${ }^{(23,24)}$ Therefore, we conducted a randomized controlled trial to compare initial hemoglobin and hematocrit levels and short term outcomes between DCC and CM among preterm infants.

\section{Methods}

This study was conducted in a single tertiary care center, Phramongkutklao Hospital, Bangkok, Thailand. Preterm infants delivered between 25 and 34 completed weeks of gestation were included when parental informed consent was obtained before delivery. Exclusion criteria included multiple pregnancies, hydrops fetalis, known major congenital anomalies, maternal bleeding from the placental previa or abruption, intention to withhold care, obstetrician's disagreement or parental refusal to participate in the study. Infants were allocated immediately before delivery to one of two groups using random permuted blocks of 4 sequentially numbered opaque sealed envelopes. Randomization was stratified by gestational age, $<30$ weeks and $>30$ weeks. For the DCC group, umbilical cord clamping was delayed for 60 seconds after delivering the infant's whole body. For the CM group, about $30 \mathrm{~cm}$ of umbilical cord was milked towards the umbilicus four times before clamping the cord. During the procedures, the infant was held at the introitus level for vaginal delivery or placed between the mother's thighs for cesarean section. A digital stopwatch was used to indicate timing of cord clamping in each delivery. Interruption of the procedure for cardiopulmonary resuscitation depended on the infant's condition and physician's decision. Subsequent management was at the consideration of the attending neonatologist. The primary outcomes were venous hematocrit and hemoglobin levels obtained within 2 hours after birth. The secondary outcomes were blood pressure, urine output, volume replacement or inotropic support for hypotension during the first 24 hours of life and blood transfusion for anemia within 28 days of age. The variables measured included hypothermia, polycythemia, peak serum bilirubin level, duration of phototherapy, duration of oxygen supplementation and morbidities including respiratory distress syndrome, patent ductus arteriosus, intraventricular hemorrhage, periventricular leukomalacia, necrotizing enterocolitis stage, late onset sepsis, chronic lung disease and retinopathy of prematurity. The sample size was calculated based on the difference in hematocrit level at $8 \%$ with type I error of 0.05 and power of 0.80 . The number of infants needed in each group totaled 23. Comparisons between the two groups were analyzed using the Mann-Whitney $U$ test for continuous data and Chi-square or Fisher's Exact test for categorical data. Data were analyzed on an intention-to-treat basis.

\section{Results}

Forty-four of 93 eligible infants were recruited in the study. Twenty-two were randomly allocated to each group (Fig 1). The median time of cord clamping in the $\mathrm{CM}$ group was 11.5 (4-43) seconds. No significant differences were observed regarding sex, gestational age, birth weight, mode of delivery, Apgar scores, requirement for resuscitation and rectal temperature between the two groups (Table 1). The initial hemoglobin and hemoglobin levels, mean blood pressure, heart rate, urine output and hypotension requiring fluid or inotropic therapy were comparable between both groups (Table 2). Peak serum bilirubin, duration of phototherapy, rate of blood transfusion due to anemia, incidence of intraventricular hemorrhage, necrotizing enterocolitis and other morbidities did not differ between the two groups (Table 3). The results did not change when performing subgroup analysis based on gestational age ( $<30,>30$ weeks). One infant in the DCC group presented asymptomatic polycythemia with a hematocrit of $66.7 \%$. One infant in the DCC group died on the $9^{\text {th }}$ day of life due to severe pneumoniaand acute kidney injury. 


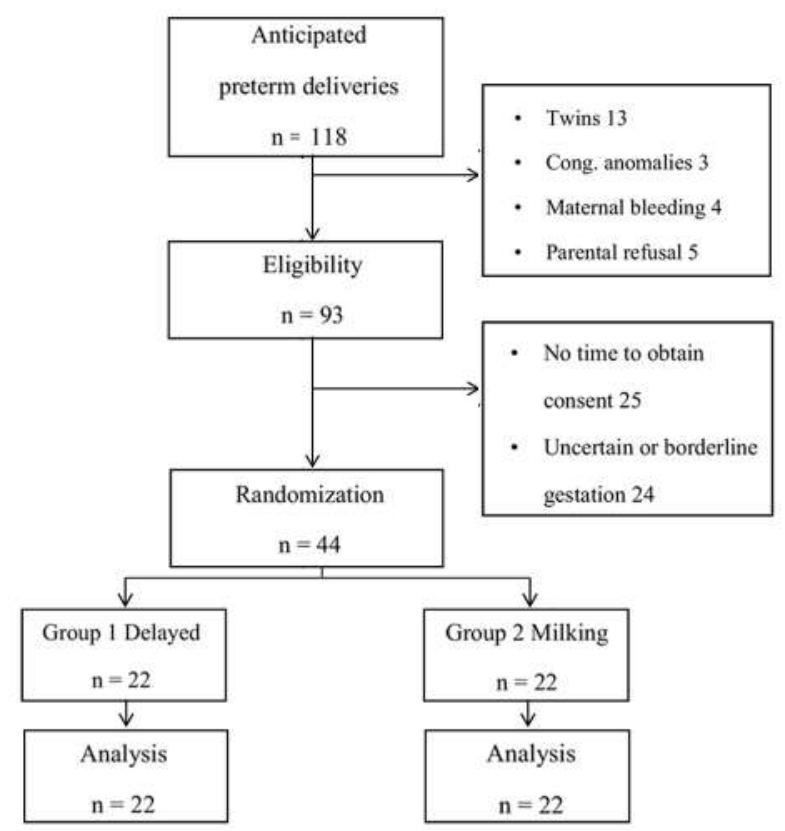

Fig 1. Flow chart of subject recruitment and analysis in the trial

Table 1. Demographic data and delivery room outcome

\begin{tabular}{lccc}
\hline & $\begin{array}{c}\text { Delayed } \\
\mathrm{n}=22\end{array}$ & $\begin{array}{c}\text { Milking } \\
\mathrm{n}=22\end{array}$ & $p$-value \\
\hline Maternal preeclampsia or chronic & $11(50.0)$ & $11(50.0)$ & 1.000 \\
hypertension (\%) & $1(4.50)$ & $4(18.2)$ & 0.345 \\
Maternal diabetes (\%) & $12(54.5)$ & $12(54.5)$ & 1.000 \\
Male gender (\%) & $32(27-34)$ & $33(26-34)$ & 0.410 \\
Gestational age (week) & $5(22.7)$ & $6(27.3)$ & \\
$\quad 17(72.3)$ & $16(72.7)$ & \\
$\quad>30$ weeks & $1724(922,2210)$ & $1766(682,2280)$ & 0.925 \\
Birth weight (gram) & $0(0.0)$ & $1(4.5)$ & 1.000 \\
Small for gestational age & $1(4.5)$ & $0(0.0)$ & 1.000 \\
Large for gestational age & $15(68.2)$ & $15(68.2)$ & 1.000 \\
Cesarean delivery $(\%)$ & $7.5(2,9)$ & $6(0,9)$ & 0.106 \\
Apgar score at 1 minute & $8(5,9)$ & $8(1,9)$ & 0.323 \\
Apgar score at 5 minutes & $0(0.0)$ & $2(9.1)$ & 0.488 \\
Apgar score $<5$ at 5 minutes $(\%)$ & $1(4.5)$ & $2(9.1)$ & 1.000 \\
Chest compression or adrenaline $(\%)$ & $36.5(35.3,37.0)$ & $36.4(35.1,37.2)$ & 0.828 \\
Delivery room temperature $\left({ }^{\circ} \mathrm{C}\right)$ & $11(50.5)$ & $12(54.5)$ & 0.763 \\
Hypothermia $(\%)$ & & & \\
\hline
\end{tabular}


Table 2. Hematocrit and hemoglobin levels and hemodynamic status within 24 hours of life

\begin{tabular}{lccc}
\hline \multicolumn{1}{c}{ Variables } & $\begin{array}{c}\text { Delayed } \\
\mathrm{n}=22\end{array}$ & $\begin{array}{c}\text { Milking } \\
\mathrm{n}=22\end{array}$ & $p$-value \\
\hline Hematocrit within 2 hours (\%) & $49.8(36.9,66.7)$ & $50.8(38.8,64.8)$ & 0.760 \\
Hemoglobin within 2 hours (g/dL) & $17.1(13.1,21.3)$ & $17.1(14.0,22.5)$ & 0.963 \\
Mean blood pressure (mmHg) & $45.7(40.0,54.3)$ & $46.0(39.7,57.3)$ & 0.817 \\
Heart rate (beats /min) & $141.7(120.7,157.3)$ & $137.7(115.0,170.3)$ & 0.337 \\
Urine output (mL/kg) & $70.2(7.1,143.5)$ & $82.7(30.6,151.8)$ & 0.253 \\
Fluid replacement $(\%)$ & $3(13.6)$ & $4(18.2)$ & 1.000 \\
Inotropic drug $(\%)$ & $2(9.1)$ & $2(9.1)$ & 1.000 \\
\hline
\end{tabular}

Table 3. Neonatal outcomes

\begin{tabular}{lccc}
\hline \multicolumn{1}{c}{ Variables } & $\begin{array}{c}\text { Delayed } \\
\mathrm{n}=22\end{array}$ & $\begin{array}{c}\text { Milking } \\
\mathrm{n}=22\end{array}$ & $p$-value \\
\hline Maximum bilirubin (mg/dL) & $8.8(6.4,12.5)$ & $8.8(3.0,15.0)$ & 0.991 \\
Duration of phototherapy (day) & $3.5(0,9)$ & $2(0,6)$ & 0.081 \\
Polycythemia (\%) & $1(4.5)$ & $0(0.0)$ & 1.000 \\
Blood transfusion within 28 days (\%) & $3(13.6)$ & $4(18.2)$ & 1.000 \\
Respiratory distress syndrome (\%) & $6(27.3)$ & $5(22.7)$ & 0.728 \\
Patent ductus arteriosus (\%) & $5(22.7)$ & $3(13.6)$ & 0.698 \\
Intraventricular hemorrhage (\%) & $1(4.5)$ & $2(9.1)$ & 1.000 \\
Necrotizing enterocolitis (\%) & $0(0.0)$ & $2(9.1)$ & 0.488 \\
Late-sepsis (\%) & $2 / 21(9.5)$ & $3 / 22(13.6)$ & 1.000 \\
Chronic lung disease (\%) & $4 / 21(19.0)$ & $4 / 22(18.2)$ & 1.000 \\
Retinopathy of prematurity requiring laser & $0 / 21(0.0)$ & $1 / 22(4.5)$ & 1.000 \\
therapy (\%) & & & \\
\hline
\end{tabular}




\section{Discussion}

Our study demonstrated that $\mathrm{CM}$ was comparable to DCC regarding the effect on initial hematologic values and hemodynamic status among preterm infants. Recently, only a few studies have compared DCC and CM among preterm infants with equivocal results. Rabe et al ${ }^{(23)}$ did not find differences in initial hematocrit, maximum serum bilirubin and morbidities between the groups. In contrast, Katheria, et al. ${ }^{(24)}$ exhibited higher delivery room temperature, initial hemoglobin, urine output and blood pressure among infants delivered by cesarean section that underwent CM.No such differences were observed in vaginally delivered infants. The disparity between these two studies was probably due to the higher rate of cesarean section among infants undergoing $\mathrm{CM}$ in Rabe et al. ${ }^{(23)}$ It has been speculated that anesthesia and surgical intervention may interfere with uterinecontraction which subsequently resulted in decreased blood flow from the placenta to the infant. Our study revealed a high rate of cesarean delivery but the rate was comparable in both groups. The method of our study differed slightly from others, e.g., length of milked cord and infant position during the procedures. The median time of cord clamping in our CM group (11.5 seconds) was almost one half of Katheria et al. (20 seconds). ${ }^{(24)}$ The shorter and varied time of $\mathrm{CM}$ in our study may have affected blood volume transferred from the placenta to the infant. One infant in the DCC group had polycythemia. She was a 32-week-gestation, 1,848 gm infant born to a mother who had chronic hypertension with superimposed pre-eclasia. Though intra-uterine growth restriction, large for gestational age and infants of diabetic mothers are important risks factors for neonatal polycythemia, we did not observe polycythemia among other infants at risk. We propose that the risks should not be contraindications for DCC or CM among preterm infants. However, further studies are warranted to determine the optimal timing for DCC and technique of $\mathrm{CM}$ in this group of infants.Our study had limitation from the inadequate power to compare complications of the procedures and neonatal morbidities between the two groups. Despite being an alternative to DCC, one concern is that CM may provide rapid bolus of blood to the infant. Moreover, the effec- tiveness of $\mathrm{CM}$ may depend on the milking technique and time of cord clamping which may vary from case to case.

\section{Conclusion}

We conclude that performing DCC or $\mathrm{CM}$ during vaginal or cesarean deliveries is feasible. Both procedures provided comparable effects on short term outcomes among preterm infants. We suggest that larger studies be undertaken to answer unclear questions. In addition, further studies on long term neuro-developmental outcomes in both DCC and $\mathrm{CM}$ groups are warranted.

\section{References}

1. Rabe H, Diaz-Rossello JL, Duley L,Dowswell T. Effect of timing of umbilical cord clamping and other strategies to influence placental transfusion at preterm birth on maternal and infant outcomes. Cochrane Database Syst Rev 2012; 8: CD003248.

2. Ultee CA, van der Deure J, Swart J,Lasham C, van Baar AL. Delayed cord clamping in preterm infants delivered at 34-36 weeks' gestation: a randomised controlled trial. Arch Dis Child Fetal Neonatal Ed 2008; 93: F20-3.

3. Oh W, Fanaroff AA, Carlo WA, DonovanEF, McDonald SA, Poole WK; Eunice Kennedy Shriver National Institute of Child Health and Human Development Neonatal Research Network. Effects of delayed cord clamping in very-low-birth-weight infants. J Perinatol 2011 (Suppl 1): S68-71.

4. Ranjit T, Nesargi S, Rao PN, Sahoo JP,Ashok C, Chandrakala BS, et al. Effect of early versus delayed cord clamping on hematological status of preterm infants at 6 wk of age. Indian J Pediatr 2015; 82: 29-34.

5. Kugelman A, Borenstein-Levin L, RiskinA, Chistyakov I, Ohel G, Gonen R, et al. Immediate versus delayed umbilical cord clamping in premature neonates born $<35$ weeks: a prospective, randomized, controlled study. Am J Perinatol 2007; 24: 307-15.

6. Meyer MP, Mildenhall L. Delayed cord clamping and blood flow in the superior vena cava in preterm infants: an observational study. Arch Dis Child Fetal Neonatal Ed 2012; 97: F484-6.

7. Sommers R, Stonestreet BS, Oh W,Laptook A, Yanowitz TD, Raker C, et al. Hemodynamic effects of delayed cord clamping in premature infants. Pediatrics 2012; 129: e667-72. 
8. Rabe H, Wacker A, Hülskamp G, Hörnig-Franz I, Schulze-Everding A, Harms E, et al. A randomised controlled trial of delayed cord clamping in very low birth weight preterm infants. Eur J Pediatr 2000; 159: 775-7.

9. Rabe H, Reynolds G, Diaz-Rossello J.A systematic review and meta-analysis of a brief delay in clamping the umbilical cord of preterm infants. Neonatology 2008; 93: 138-44.

10. Hofmeyr GJ, Bolton KD, Bowen DC,Govan JJ. Periventricular / intraventricular haemorrhage and umbilical cord clamping. Findings and hypothesis. S Afr Med J 1988; 73: 104-6.

11. Mercer JS, Vohr BR, McGrath MM,Padbury JF, Wallach $\mathrm{M}, \mathrm{Oh} \mathrm{W}$. Delayed cord clamping in very preterm infants reduces the incidence of Intraventricular hemorrhage and late-onset sepsis: a randomized, controlled trial.Pediatrics 2006; 117: 1235-42.

12. Raju TN, Singhal N. Optimal timing forclamping the umbilical cord after birth. Clin Perinatol 2012; 39: 889-900.

13. Raju TN. Timing of umbilical cordclamping after birth for optimizing placental transfusion. Curr Opin Pediatr. 2013; $25: 180-7$.

14. Hosono S, Mugishima H, Fujita H,Hosono A, Minato M, Okada T, et al. Umbilical cord milking reduces the need for red cell transfusions and improves neonatal adaptation in infants born at less than 29 weeks' gestation: a randomized controlled trial. Arch Dis Child Fetal Neonatal Ed 2008; 93: F14-9.

15. Hosono S, Mugishima H, Fujita H,Hosono A, Okada T, Takahashi S, et al. Blood pressure and urine output during the first $120 \mathrm{~h}$ of life in infants born at less than 29 weeks' gestation related to umbilical cord milking. Arch Dis Child Fetal Neonatal Ed 2009; 94: F328-31.

16. Takami T, Suganami Y, Sunohara D,Kondo A, Mizukaki N, Fujioka T, et al. Umbilical cord milking stabilizes cerebral oxygenation and perfusion in infants born before 29 weeks of gestation. J Pediatr 2012; 161: 742-7.

17. March MI, Hacker MR, Parson AW,Modest AM, de Veciana M. The effects of umbilical cord milking in extremely preterm infants: a randomized controlled trial. J Perinatol. 2013; 33: 763-7.

18. Patel S, Clark EA, Rodiguez CE, MetzTD, Abbaszadeh M, Yoder BA. Effect of umbilical cord milking on morbidity and survival in extremely low gestational age neonates. Am J Obstet Gynecol 2014; 211: 519.e1-7.

19. Katheria A, Blank D, Rich W, Finer N.Umbilical cord milking improves transition in premature infants at birth. PLoS One 2014; 9: e94085.

20. Alan S, Arsan S, Okulu E, Akin IM, KilicA, Taskin S, et al. Effects of umbilical cord milking on the need for packed red blood cell transfusions and early neonatal hemodynamic adaptation in preterm infants born $\leq$ $1500 \mathrm{~g}:$ A prospective, randomized, controlled trial. J Pediatr Hematol Oncol 2014; 36: e493-8.

21. Katheria AC, Leone TA, Woelkers D, Garey DM, Rich W, Finer NN. The effects of umbilical cord milking on hemodynamics and neonatal outcomes in premature neonates. J Pediatr 2014; 164: 1045-1050.

22. Wyckoff MH, Aziz K, Escobedo MB,Kapadia VS, Kattwinkel J, Perlman JM, et al. Part 13: Neonatal resuscitation: 2015 American Heart Association Guideline Update for Cardiopulmonary Resuscitation and Emergency Cardiovascular Care. Circulation. 2015; 132 (Suppl 2): S543-60.

23. Rabe H, Jewison A, Alvarez RF, Crook D, Stilton D, Bradley R, et al; Brighton Perinatal Study Group. Milking compared with delayed cord clamping tdo increase placental transfusion in preterm neonates: a randomized controlled trial. Obstet Gynecol. 2011; 117 : 205-11.

24. Katheria AC, Truong G, Cousins L,Oshiro B, Finer NN. Umbilical cord milking versus delayed cord clamping in preterm infants. Pediatr 2015; 136: 61-9. 